\title{
Evaluación de la calidad en el servicio: una aplicación práctica en un establecimiento de Café * $^{*}$
}

Evaluation of the Service Quality: A Practical Experience in a Coffeehouse Chain

\author{
Luis Enrique Ibarra-Morales \\ Doctor en Filosofía, Universidad Estatal de Sonora, \\ Hermosillo-México, luis.ibarra@ues.mx \\ Laura Elena Woolfolk-Gallego \\ Doctora en Ciencias, Universidad Estatal de Sonora, \\ Hermosillo-México, laura.woolfolk@ues.mx \\ Bethania Irelia Meza-López \\ Magíster en Administración, Universidad Estatal de Sonora, \\ Hermosillo-México, bethania05@hotmail.com \\ Edith Teresita Gelain-Rodríguez \\ Licenciada en Administración de Empresas, Universidad Estatal de Sonora, \\ Hermosillo-México, teresita.gelain@gmail.com
}

Cómo citar / How to cite

Ibarra-Morales, L. E., Woolfolk-Gallego, L. E., Meza-López, B. I., \& Gelain-Rodríguez, E. T. (2020). Evaluación de la calidad en el servicio: una aplicación práctica en un establecimiento de Café. Revista CEA, 6(11), 89-107. https://doi.org/10.22430/24223182.1430

Recibido: 17 de julio de 2019

Aceptado: 16 de noviembre de 2019

\section{Resumen}

La evaluación de la calidad en el servicio se ha convertido en una herramienta muy poderosa para que las organizaciones logren, entre otros propósitos, fidelizar a los clientes y ser más competitivas. En un contexto particular; este artículo ofrece un estudio de carácter cuantitativo sobre la percepción de los clientes en torno a la calidad en el servicio brindado por una cadena de café localizada en

\footnotetext{
${ }^{*}$ Este artículo se deriva del proyecto titulado «Evaluación de la calidad en el servicio. Una aplicación práctica del método Servperf en los establecimientos de café de Hermosillo, Sonora, México» y ha sido financiado con recursos propios y respaldado por la Universidad Estatal de Sonora.
} 
Hermosillo, Sonora. El objetivo general es hacer esta evaluación a través de la metodología Servperf, en los establecimientos de café de una marca reconocida. Con este objetivo, fue necesario aplicar una encuesta a 750 clientes a fin de determinar que las pruebas de validez y consistencia interna de la escala fueron satisfactorias, con un alfa de Cronbach a nivel de 0.977. Para indagar sobre la estructura subyacente de los 25 ítems que integraron la encuesta, se empleó el análisis de componentes principales con rotación Varimax, que reveló la existencia de dos variables que explican el 70.999 \% de la varianza. Como parte de los resultados principales, se concluye que la encuesta adaptada a partir de la metodología Servperf permitió evaluar de forma exitosa la calidad en el servicio otorgado por la cadena de café y el nivel de satisfacción global del cliente; así lo determinó el coeficiente de correlación Rho de Spearman, con un alto porcentaje de correlación (74.3\%) entre ambas variables. Por último, la dimensión que centró la confianza y la capacidad de respuesta fue la que obtuvo una mayor correlación (0.578), con respecto a la dimensión de empatía y tangibles (0.410).

Palabras clave: control de calidad, Servperf, satisfacción, percepción.

\title{
Clasificación JEL: M11, M32, C38.
}

\begin{abstract}
Service quality evaluation has become a very powerful tool for organizations to achieve, among other purposes, customer loyalty and competitiveness. In a particular context, this article offers a quantitative study of customer perception of the service quality provided by a coffeehouse chain located in Hermosillo, Sonora. The general objective is to evaluate the quality of the service, through the Servperf methodology, in the coffee houses of a recognized brand. Consequently, a survey of 750 clients was required to determine that the validity and internal consistency tests of the scale were satisfactory, with a Cronbach's alpha at the level of 0.977. To investigate the underlying structure of the 25 items that made up the survey, a Varimax rotation in Principal Component Analysis (PCA) was used, revealing the existence of two variables that explain $70.999 \%$ of the variance. As part of the main results, it can be concluded that the adapted survey, based on the Servperf methodology, allowed for a successful evaluation of the quality of service provided by the coffeehouse chain and the level of overall customer satisfaction, as determined by Spearman's Rho correlation coefficient, with a high percentage of correlation (74.3\%) between both variables. Finally, the confidence and responsiveness dimension had a higher correlation (0.578) than the empathy and tangible dimension (0.410).
\end{abstract}

Keywords: Quality control, Servperf, satisfaction, perception.

JEL classification: M11, M32, C38.

\section{INTRODUCCIÓN}

Hoy en día, el servicio al cliente es muy importante para las empresas, ya que, cuando se ofrece de manera positiva, se crea un vínculo de lealtad con la marca (Arifine et al., 2019). Todo dueño de un establecimiento busca fidelizar a los clientes, pues entiende que, cuando estos obtienen o hacen uso de los servicios o bienes de la empresa, puede ofrecerles el grado de satisfacción que buscan (Vargas \& Aldana, 2014; Ebrahimi et al., 2019). 
La medición de la calidad del servicio sigue siendo de interés por parte de investigadores y académicos expertos en la materia. Diferentes autores señalan los trabajos iniciados por Parasuraman et al., (1985; 1988), quienes validaron el Servqual (SERVice QUALity) como el modelo de mayor utilidad y aplicación para evaluar la calidad en los servicios. Sin embargo, Cronin y Taylor (1992; 1994), como se citó en Betancourt-Ramírez et al., (2014), «propusieron el modelo Servperf (SERVice PERFormance) (...) Mediante algunos estudios empíricos en distintas organizaciones de servicios, llegaron a la conclusión de que el modelo Servqual, propuesto por Parasuraman et al., (1985) no era el más adecuado para evaluar la calidad del servicio».

El modelo Servperf utiliza únicamente los ítems para medir la percepción como fuente de aproximación a la satisfacción del cliente, definida como «el nivel del estado de ánimo de una persona que resulta de comparar el rendimiento percibido de un producto o servicio con sus expectativas» (Kotler \& Keller, 2006, pp. 40-41; Ingaldi, 2016).

Si bien es cierto que la cadena mexicana de café se ha mantenido a través de los años en el mercado sonorense, hoy en día no está tan posicionada como en sus inicios. Pese a que México produce café, la ruta de consumidores es dominada por empresas internacionales, tanto en retail (liderada por la marca Nestlé) como en el canal de cafeterías (donde lidera la cadena Starbucks). Con la llegada de nuevos negocios y franquicias internacionales que presentan el mismo giro comercial (Olympus, The Italian Coffee, D'Volada, Café Punta del Cielo, Bendita Patria, entre otros), la cadena de café se ha debilitado en tanto que ha dejado de ser la marca preferida de los consumidores. No obstante, en cuanto a las tendencias de consumo, aunque los líderes siguen siendo internacionales (Starbucks), es evidente una proliferación de cafeterías mexicanas de cadena, así como cafeterías independientes y tostadores locales (Euromonitor Consulting, 2017).

En razón a esto, se estima que, con el paso del tiempo, la cadena de café no ha generado estrategias de mercadotecnia para atraer nuevos clientes, lo que ha provocado, en cierta medida, que las nuevas generaciones prefieran otras marcas, pues sus productos siguen siendo los mismos o no son tan creativos al lanzar un nuevo producto. Por otra parte, la creación de establecimientos de café en puntos estratégicos de la ciudad y los modelos de autoservicio o drive thru han sido una idea bastante buena, ya que facilitan la rapidez de la preparación de los productos y optimizan el tiempo de sus clientes, con lo cual se puede ofrecer un servicio rápido.

Lo anterior ofrece la problemática o disyuntiva de saber si la percepción de la calidad en el servicio varía de un cliente a otro, y si es él quien lo determina. Para conocer el nivel de percepción de los clientes en cuanto a la calidad del servicio que ofrece la cadena de café, es necesario que la empresa se esfuerce en identificar las necesidades de los clientes y determinar cuáles pueden afectar o afectan la percepción del valor de un servicio.

En ese sentido, el presente artículo tiene como propósito evaluar la calidad en el servicio mediante la percepción de los clientes de una cadena de café localizada en el municipio de Hermosillo, Sonora, a partir de la metodología Servperf, para detectar las dimensiones que ejercen mayor influencia en la satisfacción global del cliente y poder responder a las siguientes interrogantes:

— ¿Cuál es el grado de correlación que existe entre las dimensiones evaluadas y la satisfacción global del cliente? 
— ¿Cuál es el grado de correlación que existe entre las dimensiones evaluadas y la calidad en el servicio?

Para alcanzar este objetivo, se diseñó y aplicó una encuesta adaptada del método Servperf, con 25 ítems, a 750 clientes de la cadena de café, mediante una metodología de investigación de corte cuantitativo, transversal y no experimental.

Esta investigación está estructurada en los siguientes apartados: en primer lugar, la introducción, en la que se exponen de forma clara la problemática y el objetivo general del estudio, seguido del marco teórico, en este apartado se describen las diferentes definiciones y teorías en el campo de la investigación, a partir de estudios previos en otros contextos. Asimismo, se explica la metodología utilizada y se detallan el método y la estrategia de análisis, así como las técnicas estadísticas y el criterio de selección de la muestra con base en la población definida.

En el apartado de resultados, estos se describen y explican sencilla y claramente, de forma gráfica y tabular. Seguidamente, se presenta la discusión de los resultados más importantes, mediante una comparación con otros resultados similares publicados. De igual forma, se incluyen las implicaciones prácticas y teóricas del estudio. Por último, se describen las principales conclusiones derivadas de los hallazgos obtenidos.

\section{MARCO TEÓRICO}

La calidad del servicio es un concepto que surge a partir de la definición de calidad, entendida como la satisfacción de las necesidades y expectativas del cliente o, en palabras de Juran (1996), como «una aptitud de uso».

En la literatura sobre la calidad del servicio, el concepto de calidad se refiere a la calidad percibida, lo que en términos de servicio significaría «un juicio global, o actitud, relacionada con la superioridad del servicio» (Parasuraman et al., 1988). En este sentido, la calidad percibida es subjetiva, supone un nivel de abstracción más alto que cualquiera de los atributos específicos del producto y tiene una característica multidimensional (Žabkar et al., 2010). Finalmente, para Duque Oliva (2005), «la calidad percibida se valora (alta o baja) en el marco de una comparación, respecto de la excelencia o superioridad relativas de los bienes o servicios que el consumidor ve como sustitutos».

El autor en mención refiere también que Parasuraman et al., (1985) determinan tres dimensiones de la calidad, a partir de la interacción entre un cliente y los elementos de la organización de un servicio:

1) Calidad física: incluye los aspectos físicos del servicio.

2) Calidad corporativa: lo que afecta la imagen de la empresa.

3) Calidad interactiva: interacción entre el personal y el cliente, y entre clientes.

Para Mosquera-González et al., (2019), la medición de la calidad adquiere cierta trascendencia a medida que la organización es tan eficaz como para brindar un servicio de calidad. No obstante, es importante tener bien definidas las estrategias y los métodos para lograr una excelente evaluación; de lo contrario, se pueden presentar inconsistencias y limitaciones en la misma evaluación (Gulc, 2017; Calabrese \& Scoglio, 2012). 
Con base en lo anterior, la medición de la calidad se convierte en algo ineludible, ya que es necesario valorar la calidad en el servicio que se está entregando por parte de la organización, máxime cuando esta engloba factores o dimensiones que son determinantes para satisfacer a un cliente y lograr que regrese de nuevo a la empresa o, en el mejor de los casos, crear fidelidad entre la empresa y el cliente (Hemalatha et al., 2018; Gupta et al., 2018).

\section{Modelos de medición de la calidad}

Para poder medir la calidad en el servicio es importante saber qué modelos o metodologías existen y desarrollar así el estudio. A continuación, se presentan los diferentes tipos de modelos.

\section{La escuela nórdica}

Este modelo fue formulado por Grönroos (1984) y crea un vínculo entre la calidad y la imagen corporativa. Plantea que la calidad percibida por los consumidores es la integración de la calidad técnica - qué se ofrece- y la calidad funcional - cómo se ofrece-, en relación con la imagen corporativa (Fleischman et al., 2016). En este modelo, la imagen es uno de los elementos básicos para medir la percepción de la calidad que tienen los clientes.

\section{La escuela americana}

El modelo de la escuela americana de Parasuraman se ha nombrado Servqual y es el más utilizado, dada la proliferación de artículos en el área que usan su escala (Parasuraman et al., 1985). Estos autores partieron del paradigma de la desconfirmación, al igual que Grönroos, para poder desarrollar un instrumento que permitiera medir la percepción de la calidad en el servicio, para lo cual se plantean las siguientes dimensiones generales: confianza o empatía, fiabilidad, responsabilidad, capacidad de respuesta y tangibles, descritas en la tabla 1.

Tabla 1. Dimensiones del modelo Servqual

Table 1. Dimensions of the Servqual Model

\begin{tabular}{ll}
\hline \multicolumn{1}{c}{ Dimensión } & $\begin{array}{l}\text { Mide la apariencia de las instalaciones físicas, de los equipos y de los } \\
\text { empleados }\end{array}$ \\
\hline Fiabilidad & $\begin{array}{l}\text { Mide la capacidad de la organización para cumplir con lo prometido y } \\
\text { hacerlo sin errores }\end{array}$ \\
\hline Capacidad de respuesta & $\begin{array}{l}\text { Mide la voluntad de ayuda a los usuarios y la rapidez y agilidad del } \\
\text { servicio }\end{array}$ \\
\hline Responsabilidad & $\begin{array}{l}\text { Mide el conocimiento y la cortesía de los empleados y su capacidad para } \\
\text { inspirar confianza y seguridad }\end{array}$ \\
\hline Empatía & $\begin{array}{l}\text { Mide la atención esmerada e individualizada, la facilidad de acceso a la } \\
\text { información, la capacidad de escuchar y entender las necesidades }\end{array}$
\end{tabular}

Fuente: elaboración propia, a partir de (Cuellar Martín et al., 2009; Parasuraman et al., 1988), citados por Torres Fragoso y Luna Espinoza (2017). 
Para Manulik et al., (2016), el método Servqual se ha convertido en una práctica común para evaluar la calidad deseada (expectativa) en la prestación de un servicio frente a la calidad real experimentada (percepción) por los clientes al momento de recibirlo.

\section{Modelo de los tres componentes}

Está compuesto por tres elementos: el servicio y sus características, el proceso de envío o entrega y el ambiente que rodea dicho servicio. El primer elemento se refiere al diseño del servicio antes de ser entregado al consumidor: «El punto clave es la determinación de las características relevantes o especificaciones a ofrecer» (Rust \& Oliver, 1994).

\section{Servperf}

Cronin y Taylor (1992) fundamentaron una escala más concisa que el Servqual y la llamaron Servperf, método basado únicamente en la valoración de las percepciones, con una metodología similar a la anterior, que tiene en cuenta expectativas y percepciones. Así mismo, los autores afirman que la escala Servqual «no presentaba tanto apoyo teórico y evidencia empírica como punto de partida para medir la calidad de servicio percibida», en razón a lo cual, desarrollaron su propia escala tras una revisión de la literatura existente, la cual ofrece un considerable apoyo técnico.

\section{METODOLOGÍA}

El presente estudio es de carácter exploratorio. Hernández-Sampieri et al., (2014) definen los estudios exploratorios como aquellos que «se emplean cuando el objetivo consiste en examinar un tema poco estudiado o novedoso». En ese sentido, el asunto en cuestión ha sido poco explorado, al menos en el contexto actual, por lo que esta investigación tiene un valor importante en el establecimiento de puntos de referencia para futuras investigaciones y nuevas perspectivas sobre la calidad del servicio en el sector de expendios de café o cafeterías.

Asimismo, presenta un carácter correlacional y del tipo cuantitativo. Según Hernández-Sampieri et al., (2014), este tipo de estudios tienen como propósito «asociar variables mediante un patrón predecible para un grupo o población», con enfoque cuantitativo, ya que "el investigador utiliza sus diseños para analizar la certeza de las hipótesis formuladas en un contexto en particular o para aportar evidencias respecto de los lineamientos de la investigación».

En tanto que «indaga la incidencia de las modalidades, categorías o niveles de una o más variables en una población», esta investigación es de tipo descriptiva y no experimental, pues «está más cerca de las variables formuladas hipotéticamente como 'reales' y, en consecuencia, se tiene mayor validez externa (posibilidad de generalizar los resultados a otros individuos y situaciones comunes» (Hernández-Sampieri et al., 2014). Finalmente, es transversal, ya que se recolectaron datos en un solo momento, en un tiempo único, es decir, de octubre a diciembre de 2018. 


\section{Población}

La población de estudio se delimitó a aquellas personas mayores de edad (18 años) que hubieran acudido al menos una vez en la última semana, antes de aplicar la encuesta, a degustar algún tipo de café o producto en cualquiera de los 23 establecimientos ubicados en Hermosillo, Sonora, propiedad de la cadena de café.

\section{Muestra}

Para el desarrollo de esta investigación se utilizó una muestra compuesta por 750 clientes, hombres y mujeres. El muestreo fue intencional, no probabilístico, por la facilidad de acceder a los clientes in situ, con un nivel de confianza del $97 \%$ y un margen de error de $\pm 4 \%$. De acuerdo a Murray y Larry (2009), se procedió al cálculo de la muestra con (1):

$$
n=\frac{Z_{\propto}^{2} * p * q}{e^{2}}
$$

Donde:

n: tamaño de la muestra.

Z $\alpha 2$ : valor correspondiente a la distribución de gauss; $Z \alpha=0.03=2.17$.

$p$ : prevalencia esperada del parámetro a evaluar, en caso de desconocerse $(p=0.5)$, que hace mayor el tamaño de la muestra.

$q: 1-p$.

e: error que se prevé cometer.

\section{Procedimiento}

La recolección de datos se hizo por conveniencia durante un mes, en diferentes horarios de afluencia de los clientes. Con este fin, se diseñó un instrumento de medición (encuesta) con base en la escala o metodología utilizada por Servperf y se capturó y procesó en el Statistical Package for the Social Sciences (SPSS v.23 en español para Windows).

Para el diseño del conjunto de ítems que aseguraron la evaluación de la calidad del servicio de los establecimientos, se tuvo en cuenta, entre otros estudios, el de Varela Mallou et al., (2006), cuya escala fue adaptada de acuerdo a los requerimientos de la cadena de café.

El instrumento de medición constaba de 25 ítems, agrupados en las cinco dimensiones que contempla la metodología Servperf: Tangibles, Fiabilidad, Responsabilidad, Capacidad de respuesta y Empatía (Cronin \& Taylor, 1994). Para las respuestas, se empleó una escala de Likert de 6 puntos: 1) Muy mala; 2) Mala; 3) Regular; 4) Buena; 5) Muy buena; 6) Excelente, con lo cual se evitaron las respuestas neutrales.

Por último, se incluyeron dos preguntas claves: una, para evaluar el grado de satisfacción total, de acuerdo al servicio bridado, y otra, para evaluar la calidad global del servicio otorgado por el 
establecimiento. De igual manera, la encuesta incluyó preguntas relacionadas con aspectos sociodemográficos del encuestado.

\section{Análisis de datos}

Para verificar la estructura interna de la encuesta, se aplicó un análisis en componentes principales, con rotación Varimax. A fin de extraer el número de componentes, se tuvo en cuenta el modelo teórico sobre la estructura de cinco dimensiones básicas (personal, servicios, producto, instalaciones y fidelidad) (Fornieles et al., 2014).

El Alfa de Cronbach es el estadístico más ampliamente usado para probar la consistencia interna de la escala de un constructo (Cronbach, 1946). Su valor se puede encontrar en un rango entre 0 y 1 , siendo el cero una nula consistencia y el 1 una consistencia interna perfecta. De igual forma, se evaluó el promedio de correlaciones inter-ítem.

\section{RESULTADOS}

\section{Análisis de confiabilidad y validez del instrumento}

La prueba de adecuación muestral de Kaiser-Meyer-Olkin resultó satisfactoria $(\mathrm{KMO}=0.977)$ y la prueba de esfericidad de Bartlett fue estadísticamente significativa $\left(\chi 2_{(300)}=21373.349 ; p<0.001\right)$. La escala final resultante para evaluar la calidad en el servicio que se percibió incluye dos dimensiones, las cuales explican el $70.999 \%$ de la varianza total. Las escalas resultantes, tras la rotación Varimax con Kaiser, son: Responsabilidad y Capacidad de respuesta (66.424\% de la varianza explicada; 18 ítems) y Tangibles y Empatía (4.576\% de la varianza explicada, siete ítems), las cuales son definidas y mostradas en la tabla 2.

Tabla 2. Operacionalización de las variables

Table 2. Operationalization of the Variables

\begin{tabular}{ll}
\hline \multicolumn{1}{c}{ Variable } & \multicolumn{1}{c}{ Descripción } \\
\hline $\begin{array}{l}\text { Responsabilidad y Capacidad de } \\
\text { respuesta }\end{array}$ & $\begin{array}{l}\text { del personal que atiende la cafetería, como responsabilidad y capacidad } \\
\text { de respuesta al momento de atender las ordenes; incluye la amabilidad, } \\
\text { la presentación, la profesionalidad y la eficacia (Maldonado-Radillo et al., } \\
\text { 2013, p. 113). }\end{array}$ \\
\hline Se define como la percepción del usuario respecto a las condiciones \\
físicas de las instalaciones relativas a la comodidad, la decoración, el \\
mobiliario y la iluminación del local, la limpieza, el orden y equipamiento \\
de los baños y de las instalaciones, la temperatura, la ventilación y el \\
ruido; así como la percepción que el usuario tiene respecto a amplitud, \\
la variedad y la claridad de la carta, calidad, temperatura, sabor, cantidad \\
y presentación de la comida (Maldonado-Radillo, Guillén y Carranza, \\
2013, p. 113).
\end{tabular}

Fuente: elaboración propia, a partir del Análisis Factorial Confirmatorio (AFC). 
Con base en la operacionalización de las variables expuestas en la tabla 2, se formula la siguiente hipótesis general de investigación:

$\mathrm{H}_{0}$ : Las dimensiones de la calidad en el servicio no están relacionadas de forma directa y positiva con el nivel de satisfacción global del cliente.

$\mathrm{H}_{1}$ : Las dimensiones de la calidad en el servicio están relacionadas de forma directa y positiva con el nivel de satisfacción global del cliente.

Para la prueba de las hipótesis planteadas, se utilizó un modelo de regresión lineal múltiple, con la intención de mostrar el impacto (+ o -) de las dos dimensiones de la calidad en el servicio en el modelo, así como la importancia en pesos absolutos en la explicación de la variabilidad de la satisfacción global del cliente.

La fiabilidad de consistencia interna resultó aceptable, con valores de alfa de Cronbach entre 0.973 (Responsabilidad y Capacidad de respuesta) y 0.872 (Tangibles y Empatía). La correlación promedio inter-ítem resultó también adecuada para las dos escalas ( $r=0.752$ y $r=0.687$ ), lo cual es muy aceptable a valores de $r>=0.4$. (tabla 3 ).

\section{Variables sociodemográficas}

Con respecto al género, la encuesta fue respondida por 410 mujeres (54.7\%) y 340 hombres (45.3\%). El rango de edad más representativo fue el integrado por edades de 18 a 25 años (66.4\%), de los cuales, los estudiantes presentaron el mayor porcentaje, 69.7\% (523).

En cuanto al nivel de satisfacción global con respecto al género, no se encontraron diferencias estadísticamente significativas entre los grupos ( $Z=-1.696 ; p=0.090)$, es decir, hombres y mujeres se encuentran satisfechos con la calidad en el servicio otorgado por la cadena de café.

El nivel de satisfacción global tampoco fue afectado por la variable "rango de edad del encuestado»: $(x 2=6.471 ; p=0.263)$, esto es, no existen diferencias estadísticamente significativas para poder afirmar que la satisfacción global del encuestado varía entre los rangos de edad.

\section{Correlación entre la calidad en el servicio y el nivel de satisfacción global}

En la tabla 4, se expone la correlación entre las variables «calidad en el servicio» y «satisfacción global del cliente», a través del estadístico Rho de Spearman. En este punto, destaca una fuerte correlación en ambas variables, lo cual confirma aquello que la literatura y los estudios empíricos en esta materia han planteado. Este resultado responde a la pregunta de investigación número 1. 
Tabla 3. Análisis en componentes principales (rotación Varimax) y fiabilidad de consistencia interna Table 3. Principal Component Analysis (Varimax rotation) and Internal Consistency Reliability

\begin{tabular}{|c|c|}
\hline Ítems & 1 \\
\hline \multicolumn{2}{|l|}{ Factor 1. Responsabilidad y Capacidad de respuesta $(\alpha=0.973 ; r=0.752)$} \\
\hline La capacitación y competencias del personal para hacer el trabajo es... & 0.841 \\
\hline Ante solicitudes especiales de servicio, el esfuerzo extra del personal es... & 0.820 \\
\hline La atención ofrecida por el personal del establecimiento es... & 0.815 \\
\hline La relación entre la calidad de los alimentos y las bebidas con el precio es... & 0.811 \\
\hline La presentación de los alimentos y bebidas es... & 0.795 \\
\hline La capacidad de respuesta del personal que labora ante una duda o sugerencia es... & 0.782 \\
\hline La calidad de los alimentos y bebidas es... & 0.777 \\
\hline La publicidad, promociones y aplicaciones en Internet son... & 0.776 \\
\hline La oportunidad y rapidez del servicio ofrecido son... & 0.773 \\
\hline La amplitud y variedad de la carta-menú son... & 0.768 \\
\hline La cantidad servida en los alimentos y bebidas es... & 0.758 \\
\hline La presentación y atractivo de la carta-menú son... & 0.752 \\
\hline El sabor de los alimentos y bebidas es... & 0.723 \\
\hline La temperatura de los alimentos y bebidas es... & 0.711 \\
\hline El nivel de ruido del local es... & 0.693 \\
\hline La limpieza del área en donde se elaboran los alimentos y bebidas es... & 0.653 \\
\hline La limpieza y equipamiento de los baños son... & 0.538 \\
\hline Las áreas exteriores al establecimiento son... & 0.53 \\
\hline
\end{tabular}

\section{Factor 2. Tangibles y Empatía $(\alpha=0.872, r=0.687)$}

La confianza que le inspira el personal es... 
Luis Enrique Ibarra-Morales / Laura Elena Woolfolk-Gallego / Bethania Irelia Meza-López /

Edith Teresita Gelain-Rodríguez

Tabla 4. Correlación entre la calidad en el servicio y la satisfacción global

Table 4. Correlation Between Service Quality and Overall Satisfaction

\begin{tabular}{|c|c|c|c|}
\hline & & & $\begin{array}{l}\text { Nivel de satisfacción } \\
\text { global del encuestado }\end{array}$ \\
\hline \multirow{3}{*}{$\begin{array}{l}\text { Rho de } \\
\text { Spearman }\end{array}$} & & Coeficiente de correlación & $0,743^{* *}$ \\
\hline & $\begin{array}{c}\text { Evaluación global de la calidad del } \\
\text { servicio brindado por la cadena de } \\
\text { café }\end{array}$ & Sig. (bilateral) & 0,000 \\
\hline & & $\mathrm{N}$ & 750 \\
\hline
\end{tabular}

Fuente: elaboración propia, a partir de los datos procesados en el SPSS v.23.

\section{Correlación entre la calidad en el servicio y las dimensiones evaluadas}

En la tabla 5, se presenta el nivel de correlación entre las dimensiones evaluadas: Responsabilidad y Capacidad de respuesta y Tangibles y Empatía con la calidad en el servicio otorgado por los establecimientos de la cadena de café.

En este punto, sobresale que la dimensión Responsabilidad y Capacidad de respuesta fue la que tuvo una mayor correlación ( $r s=0.578, p=0.000$ ), mientras que la dimensión de Tangibles y Empatía tuvo un resultado de ( $r s=0.410, p=0.000)$. Esta información responde la pregunta de investigación número 2.

Tabla 5. Correlación entre las dimensiones y la calidad en el servicio Table 5. Correlation Between Service Dimensions and Quality

\begin{tabular}{|c|c|c|c|c|}
\hline & & & $\begin{array}{l}\text { Dimensión: } \\
\text { Responsabilidad y } \\
\text { Capacidad de } \\
\text { respuesta }\end{array}$ & $\begin{array}{l}\text { Dimensión: } \\
\text { Tangibles y } \\
\text { Empatía }\end{array}$ \\
\hline \multirow{3}{*}{$\begin{array}{l}\text { Rho de } \\
\text { Spearman }\end{array}$} & \multirow{3}{*}{$\begin{array}{l}\text { Evaluación global de la calidad del } \\
\text { servicio brindado por la cadena de } \\
\text { café }\end{array}$} & Coeficiente de correlación & $0,578^{* *}$ & $0,410^{* *}$ \\
\hline & & Sig. (bilateral) & 0,000 & 0,000 \\
\hline & & $\mathrm{N}$ & 750 & 750 \\
\hline
\end{tabular}

\section{Prueba de hipótesis general de investigación}

A continuación, se presenta el resultado para la prueba de hipótesis general de investigación formulada para el presente estudio, a través de un modelo de regresión lineal múltiple, con las variables o dimensiones involucradas en la evaluación de la calidad en el servicio, y cómo estas se relacionan con la satisfacción global del cliente de la cadena de café.

\section{Modelo de regresión lineal múltiple}

En la tabla 6, se expone el modelo general de regresión lineal de datos múltiples obtenido con las dos variables que evalúan la calidad en el servicio con el nivel de satisfacción del cliente. Se puede apreciar que el modelo explica el $45.1 \%$ de la varianza que representa el nivel de satisfacción global del cliente 
en los establecimientos de la cadena de café. De igual forma, el estadístico Durbin-Watson indica que no existen problemas de interdependencia entre las variables implicadas en el estudio.

El Anova del modelo, a través del estadístico de Fisher ( $F=307.288, p=0.000)$, confirma que el modelo no es producto del azar y se debe generalizar a la población de estudio. Por último, se confirma la relación entre las variables de estudio en un $67.2 \%$, como lo demuestra la correlación de Pearson, R.

Tabla 6. Correlación entre la calidad en el servicio y la satisfacción global Table 6. Multiple Linear Regression Model

\begin{tabular}{|c|c|c|c|c|c|c|c|}
\hline \multirow{2}{*}{ Modelo } & \multirow{2}{*}{$\mathrm{R}$} & \multirow{2}{*}{$\mathrm{R}^{2}$} & \multirow{2}{*}{$\mathrm{R}^{2}$ corregida } & \multirow{2}{*}{$\begin{array}{l}\text { Error típ. de la } \\
\text { estimación }\end{array}$} & \multirow{2}{*}{ Durbin-Watson } & \multicolumn{2}{|c|}{ Anova } \\
\hline & & & & & & $\mathrm{F}$ & Sig \\
\hline 1 & $0,672^{a}$ & 0,451 & 0,450 & 0,534 & 1,863 & 307,288 & 0,000 \\
\hline
\end{tabular}

En la tabla 7, se muestran los coeficientes de regresión para cada una de las dimensiones que evalúan la calidad en el servicio y el impacto que estos representan en la explicación de la varianza en la variable dependiente del modelo (nivel de satisfacción global). Se puede observar que, la dimensión que más impacta en la variable dependiente es Responsabilidad y Capacidad de respuesta $(\beta=0.540$, $t=19.931, p=0.000)$, seguida por la dimensión de Tangibles y Empatía $(\beta=0.400, t=14.742, p=0.000)$.

Tabla 7. Coeficientes del modelo de regresión lineal múltiple

Table 7. Coefficients of the Multiple Linear Regression Model

\begin{tabular}{ccccccc}
\hline & \multirow{2}{*}{ Modelo } & \multicolumn{2}{c}{$\begin{array}{c}\text { Coeficientes no } \\
\text { estandarizados }\end{array}$} & $\begin{array}{c}\text { Coeficientes } \\
\text { tipificados }\end{array}$ & t & Sig. \\
\cline { 2 - 7 } (Constante) & B & Error típ. & Beta & & \\
\hline Dimensión: Responsabilidad y Capacidad de respuesta & 0,389 & 0,020 & 0,540 & 19,931 & 0,000 \\
Dimensión: Tangibles y Empatía & 0,288 & 0,020 & 0,400 & 14,742 & 0,000 \\
\hline
\end{tabular}

a. Variable dependiente: Nivel de satisfacción global del cliente.

Fuente: elaboración propia, a partir de los datos procesados en el SPSS v.23.

Una vez explicado lo anterior, se prueba la hipótesis planteada en la presente investigación, para la cual se acepta la hipótesis $\left(H_{1}\right)$, ya que ambas variables o dimensiones están relacionadas de forma directa y positiva con el nivel de satisfacción global del cliente respecto a la calidad en el servicio otorgada por los establecimientos de la cadena de café.

Por último, es importante presentar la ecuación de regresión lineal múltiple que representa los coeficientes y sus betas no estandarizadas para efectos del modelo, de acuerdo a (2) y (3).

$$
\begin{gathered}
Y=\beta_{0}+\beta_{1} X_{1}+\beta_{2} X_{2}+\varepsilon \\
Y=4.132+0.389 X_{1}+0.288 X_{2}+\varepsilon
\end{gathered}
$$


Donde:

$\mathrm{Y}=$ Variable dependiente (Satisfacción global del consumidor)

$\beta_{0}=$ Constante del modelo

$\beta_{1}$ y $\beta_{2}=$ Betas del modelo

$\mathrm{X}_{1}=$ Responsabilidad y Capacidad de respuesta

$\mathrm{X}_{2}=$ Tangibles y Empatía

$\varepsilon=$ Error del modelo

\section{DISCUSIÓN}

El presente estudio tiene su justificación en el interés por evaluar la calidad en el servicio y el nivel de satisfacción global de los clientes de una cadena de café reconocida en el municipio de Hermosillo, Sonora. Con este objetivo, era importante establecer las dimensiones que impactan directamente en la satisfacción del cliente y si estas pueden determinar el nivel de calidad del establecimiento de café en la localidad. Este argumento, junto a la gran cantidad de establecimientos que ya operan en la ciudad y que son competencia directa e indirecta en algunos casos, hacen que los temas de calidad en el servicio y la satisfacción del cliente, con miras de buscar su fidelización, sean críticos al momento de instaurar estrategias para buscar una posición competitiva primordial en el sector.

La percepción del usuario respecto al personal que atiende la cafetería, en términos de responsabilidad y capacidad de respuesta al momento de surtir las órdenes (incluidas la amabilidad, la presentación, la profesionalidad y la eficacia), son los aspectos que más valoran de forma positiva. Estos resultados son similares a los estudios realizados por Salvador (2005) y Maldonado-Radillo et al., (2013), ya que reflejan la importancia que el usuario concede al trato ofrecido por los empleados de la cafetería.

El análisis de regresión lineal múltiple indica que la satisfacción global del consumidor se puede predecir a través del mismo, ya que se obtuvo un $p$-valor $<0,05$. Adicionalmente, el coeficiente de correlación R es igual a 0.672, y una R2 igual a 0.451, valores muy semejantes reportados en el estudio de Cárdenas Herrera y Gordón Cabezas (2016), en el cual se midió la satisfacción del cliente en una cafetería, a través de ocho factores, de los cuales, únicamente tres resultaron ser estadísticamente significativos y la calidad en el servicio es uno de ellos.

Así mismo, se encontraron correlaciones aceptables entre las dos dimensiones analizadas a nivel de p-valor<0.05: calidad del servicio y satisfacción general del consumidor en los establecimientos de café, como lo evidenciaron Vera y Trujillo (2009), en un análisis del que se infiere que los clientes de los establecimientos de alimentos y bebidas se sienten satisfechos y tienden a formar su opinión general sobre la calidad del servicio según la calidad de la comida y de las propias instalaciones.

Aun cuando la literatura sobre calidad del servicio argumenta que la dimensión de tangibles es considerada como menos crítica por los clientes que evalúan los servicios prestados, en esta investigación, se obtuvo una correlación Rho de Spearman por el orden de 0.410 , significativa a pvalor $<0.01$ bilateral. Lo anterior es una particularidad del servicio que el cliente espera obtener a 
partir de los elementos tangibles y que incide de forma puntual en la satisfacción global del consumidor.

En cuanto a la hipótesis de investigación planteada, se ha confirmado que existe una relación directa y positiva entre las dimensiones que evalúan la calidad en el servicio y el nivel de satisfacción global del consumidor de café. Se encontró evidencia suficiente de que la calidad en el servicio es un antecedente importante de la satisfacción, sobre la que ejerce un nivel de correlación positiva y estadísticamente significativa de 0.743 . Estos resultados pueden ser contrastados con los obtenidos por Sanmiguel Jaimes et al., (2015).

Las variables sociodemográficas estudiadas respecto al nivel de satisfacción no representaron alguna diferencia estadísticamente significativa, que pudiera hacer pensar que, en este sector particular, las personas que pertenecen a un determinado género y rango de edad perciban de forma diferente la calidad en el servicio otorgado en relación con su nivel de satisfacción global. Esto parece corroborar que, independientemente de las características de los clientes, todos los segmentos sociodemográficos exponen un nivel de satisfacción similar y las puntuaciones son uniformes o iguales.

Para la determinación de la estructura factorial de la escala de medición de la calidad que es percibida por los clientes, en primer lugar, se evaluó la consistencia interna del instrumento de medición y la fiabilidad de los ítems que lo conforman, a través del coeficiente alfa de Cronbach. Este coeficiente, en particular, alcanzó valores entre 0,973 para la dimensión: responsabilidad y capacidad de respuesta y 0.872 para la dimensión: tangibles y empatía, claramente superior al valor de 0.080 recomendado por diversos autores, entre los que destacan Patterson y Johnson (1993), Luque (2017), George y Mallery (2003). Este resultado se puede interpretar como garantía de alta fiabilidad; esto es, existe una alta homogeneidad y equivalencia en las respuestas. Se trata, pues, de un instrumento que posee un índice de consistencia interna satisfactoria acorde a lo planteado por Zapata Rotundo et al., (2008) y muy cercano al valor reportado por Varela et al., (2006).

De conformidad con estudios anteriores, el resultado también indicó una alta correlación entre las variables que miden la calidad en el servicio y la satisfacción del cliente (Jin et al., 2013; Ma et al., 2014; Mittal y Kamakura, 2001; Ramanathan et al., 2016). Como ya se mencionó, dicha relación fue estudiada por los autores Parasuraman et al., (1991), Cronin y Taylor (1992) y Colmenares y Saavedra (2007), quienes demostraron que la calidad en el servicio es un antecedente de la satisfacción de los consumidores. Así, se confirma que existe una relación directa entre la calidad en el servicio otorgado por los establecimientos de café y la satisfacción global de los consumidores, ya que, en el nivel de correlación encontrado a través del estadístico Rho de Spearman, se pudo constatar un fuerte vínculo entre ambas variables.

\section{CONCLUSIONES}

Los atributos propuestos para evaluar la calidad en el servicio en los establecimientos de café para una cadena nacional reconocida han sido adecuados para tal propósito, así lo demostraron los altos niveles de fiabilidad y consistencia interna (validez). En este sentido, se han encontrado evidencias 
empíricas y teóricas suficientes de la existencia de múltiples dimensiones para evaluar la calidad en el servicio, que, para este caso en particular, son dos.

En conclusión, este estudio arroja que las dimensiones Responsabilidad y Capacidad de respuesta, así como Tangibles y Empatía, tienen una relación significativa con la satisfacción global del cliente. De igual forma, la calidad en el servicio ofrecido por la cadena de establecimientos de café tiene una alta incidencia de correlación con la satisfacción total del consumidor.

Aunado a lo anterior, como en todo estudio empírico, existen algunas limitaciones. En primer lugar, no se puede generalizar a toda la población del estado de Sonora, ya que solo se enfocó en una ciudad, si bien es cierto, la más importante del Estado. Por lo tanto, se sugiere incorporar estudios en otras ciudades del estado de Sonora, de las cuales se podrían seleccionar aquellas que aglomeran a más de 150000 habitantes, con lo que se obtendría un estudio de investigación representativa para todo un Estado. En segundo lugar, el estudio se centró en 23 establecimientos, el muestreo fue no probabilístico por conveniencia y no por estrato con afijación proporcional, por la facilidad de acceso a los clientes por establecimiento y frecuencia de visita.

\section{Implicaciones teóricas}

Desde el punto de vista académico, el uso de ecuaciones de regresión lineal con múltiples datos o variables permite a la academia conocer mejor la influencia que ejercen las variables independientes sobre la variable que se quiere explicar y determinar el orden de importancia e impacto sobre la misma variable dependiente. En este caso, se puede conocer el grado de influencia de las dimensiones que evalúan la calidad en el servicio sobre la satisfacción global, ya que los modelos teóricos indican que dichas dimensiones ejercen un dominio positivo en esta última.

Del mismo modo, fue posible constatar que las dimensiones de la calidad en el servicio no son las únicas que determinan la satisfacción del cliente o consumidor, pues solo explican el $45.1 \%$ de la variabilidad, por lo que resulta importante considerar otras variables o factores críticos en busca de una explicación mayor, de acuerdo a la literatura y estudios empíricos previos.

\section{Implicaciones prácticas}

El instrumento Servperf, modificado a partir de Maldonado-Radillo et al., (2013), resultó ser de utilidad para la presente investigación, ya que los gerentes o administradores de los establecimientos de café de la ciudad pudieron obtener información útil sobre la percepción de la calidad en el servicio por parte de los clientes. Asimismo, permitió identificar áreas de oportunidad y proponer mejoras sustanciales.

De acuerdo a los resultados obtenidos, se deben diseñar estrategias para que los expendios de café sean mejores en todos los aspectos relacionados con el producto, las instalaciones y la innovación en el servicio ofrecido por el personal de cada uno de los 23 establecimientos de la cadena nacional de café, distribuidos en el municipio de Hermosillo, Sonora. Además, es importante buscar alternativas para brindar un mejor servicio, más ágil, rápido y oportuno, que haga del tiempo de espera un momento más agradable y satisfactorio frente a la calidad y el precio que se paga por el producto. 


\section{AGRADECIMIENTOS}

De forma puntual, se agradece a todos los usuarios que respondieron la encuesta de satisfacción y a la cadena de café, que permitió que se aplicara el instrumento de medición en sus instalaciones y se publicaran los resultados, bajo la confidencialidad de la información de la firma.

\section{REFERENCIAS}

Arifine, G., Felix, R., \& Furrer, O. (2019). Multi-brand loyalty in consumer markets: a qualitativelydriven mixed methods approach. European Journal of Marketing, 53(11), 2419-2450. https://doi.org/10.1108/EJM-07-2017-0474

Betancourt-Ramírez, J.B.; Aldana-de-Vega, L. y Gómez-Betancourt, G. (2014). Servicio, ambiente y calidad de restaurantes en Bogotá. Estudio comparativo de empresa familiar y empresa no familiar. Entramado, 10(2), 60-74. URL

Calabrese, A., \& Scoglio, F. (2012). Reframing the past: A new approach in service quality assessment. Total Quality Management \& Business Excellence, 23(11-12), 1329-1343. https://doi.org/10.1080/14783363.2012.733259

Cárdenas Herrera, R. E. \& Gordón Cabezas, A. C. (2016). Estudio del nivel de satisfacción y planteamiento de estrategias para la Cafetería "No Sea Malito" y La Pirámide a través de DINESERV y Matriz IPA. (Tesis de pregrado). URL

Colmenares, O. A. \& Saavedra, J. L. (2007). Aproximación teórica de los modelos conceptuales de la calidad del servicio. Técnica Administrativa, 6(4). URL

Cronbach, L. J. (1946). Response sets and test validity. Educational and Psychological Measurement, 6(4), 475-494. https://doi.org/10.1177/001316444600600405

Cronin, J. J., \& Taylor, S. A. (1992). Measuring service quality: A reexamination and extension. Journal of Marketing, 56(3), 55-68. https://doi.org/10.2307/1252296

Cronin, J. J., \& Taylor, S. A. (1994). SERVPERF versus SERVQUAL: Reconciling performance based and perceptions minus expectations measurement of service quality. Journal of Marketing, 58(1), 125-131. https://doi.org/10.2307/1252256

Duque Oliva, E. J. (2005). Revisión del concepto de calidad del servicio y sus modelos de medición. Innovar Revista de Ciencias Administrativas y Sociales, 15(25), 64-80. URL

Ebrahimi, L., Mirabi, V. R., Ranjbar, M. H., \& Pour, E. H. (2019). A Customer Loyalty Model for ECommerce Recommendation Systems. Journal of Information \& Knowledge Management, 18(3), p.1950036. https://doi.org/10.1142/S0219649219500369 
Euromonitor Consulting. (2017). Análisis del Mercado de Consumo de Café en México 2016. Informe del estudio realizado por Euromonitor International para AMECAFE. URL

Fleischman, G. M., Johnson, E. N., \& Walker, K. B. (2016). An Exploratory Examination of Management Accounting Service and Information Quality. Journal of Management Accounting Research, 29(2), 11-31. https://doi.org/10.2308/imar-51614

Fornieles, A., Penelo, E., Berbel, G. \& Prat, R. (2014). Escala para la calidad de los servicios y lealtad del consumidor en tiendas de alimentación. Universitas Psychologica, 13(3), 15-24. http://dx.doi.org/10.11144/Javeriana.UPSY13-3.ecs/

George, D., \& Mallery, P. (2003). SPSS for Windows step by step: A Simple Guide and Reference, 11.0 Update (4a Ed.). Allyn \& Bacon.

Grönroos, C. (1984). A service quality model and its marketing implications. European Journal of Marketing, 18(4), 36-44. https://doi.org/10.1108/EUM0000000004784

Gulc, A. (2017). Models and methods of measuring the quality of logistic service. Procedia Engineering, 182, 255-264. https://doi.org/10.1016/j.proeng.2017.03.187

Gupta, S., Gupta, T., \& Shainesh, G. (2018). Navigating from programme loyalty to company loyalty. IIMB Management Review, 30(3), 196-206. https://doi.org/10.1016/j.iimb.2018.01.009

Hemalatha, S., Dumpala, L., \& Balakrishna, B. (2018). Service quality evaluation and ranking of container terminal operators through hybrid multi-criteria decision making methods. The Asian Journal of Shipping and Logistics, 34(2), 137-144. https://doi.org/10.1016/j.ajsl.2018.06.010

Hernández-Sampieri, R., Fernández-Collado, C. \& Baptista Lucio, P. (2014). Metodología de la Investigación. (6a Ed.). McGraw-Hill.

Ingaldi, K. M. (2016). Use of the SERVPERF method to evaluate service quality in the transport company. Independent Journal of Management \& Production, 7(1), 168-177. https://doi.org/10.14807/ijmp.v7i1.396

Jin, N. P., Line, N. D., \& Goh, B. (2013). Experiential value, relationship quality, and customer loyalty in full-service restaurants: the moderating role of gender. Journal of Hospitality Marketing \& Management, 22(7), 679-700. https://doi.org/10.1080/19368623.2013.723799

Juran, J. M. (1996). Juran y la calidad por el diseño. URL

Kotler, P. \& Keller, K. L. (2006). Dirección de Marketing. (12ª Ed.). Pearson Educación.

Luque, T. (2017). Investigación de marketing 3.0. Ariel. 
Ma, E., Qu, H., \& Eliwa. R. A. (2014). Customer loyalty with fine dining: The moderating role of gender. Journal of Hospitality Marketing \& Management, 23(5), 513-535. https://doi.org/10.1080/19368623.2013.835250

Maldonado-Radillo, S. E., Guillén-Jiménez, A. M. \& Carranza-Prieto, R. E. (2013). Factores determinantes de la calidad del servicio de una cafetería en el campus de una universidad pública. Revista Internacional Administración \& Finanzas, 6(1), 109-118. URL

Manulik, S., Rosińczuk, J., \& Karniej, P. (2016). Evaluation of health care service quality in Poland with the use of SERVQUAL method at the specialist ambulatory health care center. Patient Preference and Adherence, 10(1), 1435-1442. https://doi.org/10.2147/PPA.S108252

Mittal, V. \& Kamakura, W. A. (2001). Satisfaction, repurchase intent, and repurchase behavior: Investigating the moderating effect of customer characteristics. Journal of Marketing Research, 38(1), 131-142. https://doi.org/10.1509/jmkr.38.1.131.18832

Mosquera-González, D., Patiño-Toro, O. N., Sánchez-Díez, D. M., Agudelo-Cardona, J. F., OspinaMazo, D. M., \& Bermúdez-Bedoya, J. F. (2019). Factores asociados a la calidad en el servicio en Centros de Acondicionamiento Físico a partir del modelo SERVQUAL. Revista CEA, 5(9), 13-32. https://doi.org/10.22430/24223182.1253

Murray R. S. \& Larry, J. S. (2009). Estadística. (4a Ed.). McGraw-Hill.

Parasuraman, A., Zeithaml, V. A. \& Berry, L. (1985). A conceptual model of service quality and its implications for further research. Journal of Marketing, 49(4), 41-50. https://doi.org/10.2307/1251430

Parasuraman, A., Zeithaml, V. A. \& Berry, L. (1988). SERVQUAL: A Multiple-It Scale for Measuring Customer Perceptions of Service Quality. Journal of Retailing, 64(1), 12-40.

Parasuraman, A., Berry, L. L., Zeithaml, V. A. \& (1991). Refinement and Reassessment of the Servqual Scale. Journal of Retailing, 67(4), 420-450. $\underline{\text { URL }}$

Patterson, P. G. \& Johnson, L. W. (1993). Disconfirmation of expectations and the gap model of service quality: an integrated paradigm. Journal of Consumer Satisfaction, Dissatisfaction and Complaining Behavior, (6), 90-99. URL

Ramanathan, R., Di, Y. \& Ramanathan, U. (2016). Moderating roles of customer characteristics on the link between service factors and satisfaction in a buffet restaurant. Benchmarking: An International Journal, 23(2), 469-486. https://doi.org/10.1108/BIJ-01-2015-0012

Rust, R. T. \& Oliver, R. L. (1994). Service Quality. New Directions in Theory and Practice. http://dx.doi.org/10.4135/9781452229102

Salvador, C. M. (2005). Percepción de las dimensiones de la calidad de servicios en una muestra de usuarios españoles y paraguayos. Boletín de Psicología, 83, 69-80. URL 
Sanmiguel Jaimes, E. M.; Rivera Franco, M. X., Mancilla Medina, N. O. \& Ballesteros Monsalve, H. (2015). Medición de la calidad percibida en el servicio mediante la herramienta SERVQUAL en tiendas de café en Santander, Colombia. Criterio Libre, 13(23), 145-164. https://doi.org/10.18041/1900-0642/criteriolibre.2015v13n23.106

Torres Fragoso, J \& Luna Espinoza, I. (2017). Assessment of banking service quality perception using the SERVPERF model. Contaduría y Administración, 62(4), 1270-1293. http://dx.doi.org/10.1016/j.cya.2017.06.011

Varela Mallou, J., Prat Santaolària, R., Voces López, C. \& Rial Boubeta, A. (2006). Una nueva escala para la evaluación de la calidad de los servicios de hostelería. Psicothema, 18(1), 135-142. URL

Vargas, M. E. \& Aldana de Vega, L. (2014). Calidad y servicio. Conceptos y herramientas. (3a Ed.). URL

Vera, J. y Trujillo, A. (2009). El papel de la calidad del servicio como antecedente de la lealtad del cliente. Panorama económico, 27(38), 16-30.

Zapata Rotundo, G., Canet Giner, M. \& Peris Bonet, F. (2008). Visión directiva y elección estratégica: su influencia en los tipos básicos de trabajo. Cuadernos de Administración, 21(37), 185-209. URL

Žabkar, V., Brenčič, M. M., \& Dmitrović, T. (2010). Modelling perceived quality, visitor satisfaction and behavioural intentions at the destination level. Tourism Management, 31(4), 537-546. https://doi.org/10.1016/i.tourman.2009.06.005 